\title{
Efficacy of an Inexpensive Regimen Containing Pasiniazine for Multidrug-Resistant Tuberculosis with Low-concentration resistance to Isoniazid: a prospective cohort study in a high Tuberculosis burden area
}

\section{Wenwen Sun}

Tongji University Affiliated Shanghai Pulmonary Hospital

\section{Qin Tang}

Tongji University Affiliated Shanghai Pulmonary Hospital

Jie Wang

Tongji University Affiliated Shanghai Pulmonary Hospital

Jinghui Yang

Tongji University Affiliated Shanghai Pulmonary Hospital

\section{Fangyou Yu}

Tongji University Affiliated Shanghai Pulmonary Hospital

\section{Hua Yang}

Tongji University Affiliated Shanghai Pulmonary Hospital

Lin Fan ( $\square$ fanlinsj@163.com )

Shanghai Pulmonary Hospital, Tongji University School of Medicine https://orcid.org/0000-0002-9411496X

\section{Research Article}

Keywords: MDR-TB, inexpensive regimen, MIC, Treatment outcome, Adverse effects

Posted Date: January 15th, 2021

DOI: https://doi.org/10.21203/rs.3.rs-143795/v1

License: (c) (i) This work is licensed under a Creative Commons Attribution 4.0 International License. Read Full License 


\section{Abstract}

\section{Background}

To verify the efficacy and safety of an inexpensive regimen for specific multidrug-resistant tuberculosis (MDR-TB) based on Minimum Inhibitory Concentration (MIC) Drug sensitivity test(DST), a prospective cohort study was conducted in China.

Methods

MDR-TB cases were prospectively enrolled through a clinical access programmes with strict inclusion and exclusion criteria, given the regimen and followed up, observed the treatment outcome and adverse effects (AEs) .

Results

A total of 114 patients were enrolled into the study. The overall treatment favorable rate was $79.8 \%$ (91/114), among 91 cases with favorable treatment, 86(75.4\%)cured and $5(4.4 \%)$ completed treatment. 23 cases got unfavorable outcome including $108.8 \%$ (10/114) failures, 8.8\%(10/114) losing follow up , $0.9 \%(1 / 114)$ withdrawing treatment due to intolerance to drugs and $1.8 \%(2 / 114)$ died,respectively. Treatment favorable rate was significantly higher in newly treated MDR-TB $(91.7 \%, 33 / 36)$ than that in retreated MDR-TB $(74.4 \%, 58 / 78, p=0.03)$. The investigators recorded 42 AEs occurrences in 30 of 114 patients $₫ 26.3 \%)$. Clinicians rated most $A E s$ as mild or moderate $(95.24 \%, 40 / 42)$ and no one was caused by $\mathrm{Pa} \otimes 0 \%, 0 / 114)$.

\section{Conclusions}

The regimen was proved to be effective, safe and inexpensive. It is suitable for specific drug resistant population in China, especially for newly-treated patients, which could be expected to be developed into a short-course therapy.

Clinical Trials Registration:

China Clinical Trial Registry ChiCTR- OPC-16009380

\section{Introduction}

Multidrug-resistant tuberculosis (MDR-TB) is a major global health problem with recovery rate less than $60 \%$ (1). The 2019 WHO consolidated guidelines offered three possible therapeutic options》all-oral long/short or injection included $₫$ for countries and programs treating people with MDR-TB (1). However, it is unclear to national programmes and their implementing partners which programmes should be given priority. Treatment success rates and preferred regimens vary from country to country, depending on different factors such as financial support, management, protocol, drug quality, newly included drugs, 
patient compliance and tolerance. Therefore, how to achieve the best treatment outcome for MDR-TB and ensure its high safety has become the most important issue for clinicians. The all-oral regimen containing bedaquiline (BDQ) instead of injectable agent recommended in 2019 guideline (2) may be effective but difficult to promote in resource-poor areas due to its high price. Scholars pointed out that the explicit recommendations for the use of Linazolamide (LZD) and BDQ may also bring some disadvantages to the global TB control program (3). Ideally, MDR-TB treatment should be effective区have few adverse events囚used cheaply, easily available and drugs affordable (4).

According to the WHO 2019 report, the treatment coverage rate of MDR-TB in China was only 13.6\% (1). Delayed DST, unaffordable treatment costs and adverse drug reactions were the main reasons for the low treatment coverage(5). The WHO guideline in 2016 recommended a short-range regimen for "simple drug resistance $\$ without second-line drug resistance $\ M D R-T B$ that has proved effective in multiple countries, called "Bangladesh programme" $(6,7)$. On the other hand, MDR-TB had multiple factors which can influence treatment outcome, such,as individual treatment history (8). Therefore, we designed a treatment regimen similar to the regimen recommended by the $2016 \mathrm{WHO}$ guidelines according to national conditions and individual Drug sensitivity test $\llbracket \mathrm{DST}$ ).

Pasiniazine (Pa) is a chemical synthesis of isoniazid (INH) and paminosalicylic acid (PAS). PAS effectively delays and blocks the acetylation of INH in vivo. This product maintained a high, prolonged concentration of INH in the blood and reduces toxicity to the liver. It not only enhances the bactericidal action of the drug, but also delays the generation of bacterial resistance.

In present study, a prospective clinical trial was conducted in a national TB specialist hospital in China to evaluate the safety and efficacy of a Pa containing regimen in MDR-TB patients with low resistance of $\mathrm{INH}$ and no second-line drug/injection resistance. We hope to explore a relatively inexpensive and effective regimen for MDR-TB patients with specific drug resistance characteristics. The results of this study may influence the standard treatment model for MDR-TB in this country - individualized stratified treatment based on DST.

\section{Methods}

\section{Study design}

This study is an open-label, prospective cohort study. All patients received treatment for 18 months. They were followed up for at least one year after end of the course. The trial was conducted at a national TB clinical treatment center with nearly 300 beds receiving referral of patients with MDR-TB from eastern China. In addition, the hospital is the only specialized hospital receiving MDR-TB in Shanghai and undertakes the region formulation and management of MDR-TB programmes combined with center for

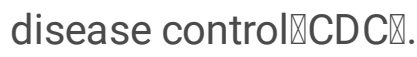

\section{Study patients}


From January 2017 to January 2018囚 we enrolled patients between 18 and 65 years diagnosed as MDRTB confirmed by BACTEC MGIT 960 liquid culture『MGIT 960囚 and Minimum Inhibitory Concentration \MIC凶DST.

Inclusion criteria were to include MDR-TB patients confirmed by MGIT960 and MIC DST at least resistant to $\mathrm{INH}$ at low concentration resistance and rifampicin $(\mathrm{R})$ resistance within 2 months prior to screening; and patients had no second-line drugs resistance including injectable agents; and patients previously only received first-line anti-TB treatment (ATT) or had no previous history of ATT or previous history of less than one month of second-line ATT.

Excluded criteria were as follows: 1) XDR-TB patients (resistant to both flquinolones and second-line injection drugs) or pre-XDR (resistant to either flquinolones or second-line injection drugs) 2 ) patients had poor compliance to any drugs within the regimens; 3 ) any serious systemic disease or disease of the immune system; 4) coexisted with extrapulmonary tuberculosis; 5) co-infected with HIV and other virus, taking immunosuppressive agents; 6 ) history of flquinolone use for more than one month in the recent 6 months.

We defined the newly diagnosis of MDR-TB as having never been treated for TB or less than one month of treatment history (9) ; Re-treated cases were defined as registered MDR-TB patients who had previously taken first-line anti-TB drugs for 1 month or more at the time of history inquiry; or if there is sufficient documentary evidence of having been treated with first-line anti-TB drugs for one month or more in the past.(10)

\section{Inclusion and intervention}

Patients satisfied with included criteria were administered by the regimen including Amikacin (Ak), Fluoroquinolones $\mathbb{F Q} \mathbb{Q} \otimes$, Cycloserine(Cs), Protionamide (Pto), Pyrazinamide(PZA) and $\mathrm{Pa}$ for six months with injectable Ak followed by 12 months of oral FQs, Cs, Pto, PZA and Pa.

All patients were screened within one week of receiving the first dose and administered under directly observed therapy (DOT) throughout the treatment course. During the course of treatment, patients were visited by the same specialist every two weeks until the end of the course. Visits were made every 3 months after completion of treatment until 12 months after completion of treatment.

\section{Microbiological assessment}

Sputum/bronchoalveolar lavage samples were collected for smear microscopy and MTB culture at the following times: at baseline; Follow-up was followed up at 1, 2, and 3 months, and then once every 3 months until the end of the course. All MTB positive isolates were transferred to the TB basic laboratory for the determination of MIC were $1 \mu \mathrm{g} / \mathrm{mL}$ for $\mathrm{Pa}, 8 \mu \mathrm{g} / \mathrm{mL}$ for $A \mathrm{k} \otimes 1 \mu \mathrm{g} / \mathrm{mL}$ for Rifampicin $\nabla R \rrbracket, 0.2 \mu \mathrm{g} / \mathrm{mL}$ for $\mathrm{INH}, 0.5 \mu \mathrm{g} / \mathrm{mL}$ for $\mathrm{Mfx}$ and $1 \mu \mathrm{g} / \mathrm{mL}$ for $\mathrm{LfX}$.

\section{Treatment efficacy evaluation}


Treatment outcomes for MDR-TB were evaluated according to WHO guidelines (11). The patients were examined by Chest CT imaging every three months and sputum culture once a month during the treatment period.

The treatment outcomes were divided into "cured" $\nabla$ "completed treatment" $\nabla$ “failure" $\nabla$ "default"and "death". The "cured" was referred as patients completed treatment with consistently at least five negative culture results for the final 12 months of the treatment course and without evidence of treatment failure(12); Completed treatment was determined by bacterial negative conversion at the end of the treatment with less than three negative cultures. The "failure" was referred as patients had sputum culture positive in the final 12 months of the treatment course or if any one of the final three cultures was positive or to be discontinued due to clinical or radiological adverse reactions or adverse events. "Death" was patients died from any reason during the course of ATT; "Default" was referred as patients whose TB treatment was interrupted for at least two consecutive months for any reason. Treatment favorable was defined as sum of cured and treatment completed , unfavorable outcomes included "failure", "default" and "death"(13).

\section{Safety assessment}

Included patients were monitored by blood and urine routine, liver function, renal function at least once a months, hearing test once a month, followed at outpatients department of TB by physicians once a

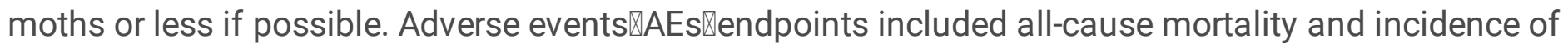
adverse events that occurred or worsened during treatment, defined by the Division of Microbiology and Infectious Diseases(14). And the severity of AEs according to whether they were determined by researchers to be related to the drug in question.

\section{Statistical analysis}

Statistical analysis was used SPSS 18.0 (IBM Corp, Armonk, NY, USA). Categorical variables such as treatment outcome, sputum conversion rate were analysed using $X^{2}$ tests and Fisher's exact tests, and continuous variables were analysed using independent t-tests and Mann-Whitney U-tests. P value $<0.05$ was considered statistically significant.

\section{Results}

\section{Study population}

During the study period, a total of 114 patients were included in the study, including 72 males with median age at 35.7 years (range19-64 years) and 42 females with median age at 32.2 years (range 19-61 years), 36 cases with newly treated MDR-TB and 78 cases with retreated MDR-TB. The general characteristics of included patients was shown in Table 1

Table 1 Clinical characteristics of patients enrolled

\section{Treatment outcome of included patients}


The overall favorable treatment rate was 79.8\% (91/114): 75.4\% (86/114) cured, 4.4\% (5/114) completed treatment. $20.2 \% \varangle 23 / 114 \llbracket$ got unfavorable outcome $₫ 8.8 \%$ (10/114) failures, $9.6 \%$ (11/114) default including $8.8 \%(10 / 114)$ losing follow up and $0.9 \%(1 / 114)$ withdrawing treatment due to intolerance to drugs; and $1.8 \%$ (2/114) died. The flow diagram of patients included was shown in Figure 1

Fig 1 Flow diagram

Treatment favorable rate was significantly higher in newly treated MDR-TB $(91.7 \%, 33 / 36)$ than that in retreated MDR-TB ( $74.4 \% \bigotimes 58 / 78) \llbracket p$ value was 0.03 . Sputum culture negative conversion rates at the end of third month and the sixth month were $91.7 \%$ (33/36) and 94.4\% (34/36) in newly treated group which were significantly higher than that of $69.2 \%(54 / 78)$ and $70.5 \%$ (55/78) in retreated group, p value was 0.009 and 0.004 , respectively. A total of $51(44.73 \%, 51 / 114)$ patients achieved the standard of stopping medication (11) at the end of the 12th month: among which $26(72 \%, 26 / 36)$ patients were newly treated, and $25(32 \%, 25 / 78)$ were retreated, $p$ value was 0.00 . The curve outcome was shown in figure 2 and Table 2.

All patients who completed the course of treatment were followed for 1 year and no recurrence was found.

Figure2 Treatment outcome

Table 2 The differences of time to culture conversion between two groups

\section{Safety and side effect monitoring $\square A d v e r s e ~ e v e n t s \square A E s \square$}

The investigators recorded 42 AEs occurrences in 30 of 114 patients $₫ 30 / 114,26.3 \%)$. Clinicians rated most $\mathrm{AEs}$ as mild or moderate ( $\mathrm{n}=40 \mathrm{AEs}, 40 / 42)$ and no one was caused by $\mathrm{Pa} \mathrm{\otimes} 0 / 114,0 \%)$.

Among them, 22 AEs occurrences in 15 patients $(13.16 \%, 15 / 114)$ experienced a change in treatment regimen. Among them, $12 \mathrm{AEs}$ occurrence in 10 patients associated with Ak due to mild hearing loss $(n=5)$ or mild renal dysfunction $(n=7) .6$ AEs occurrences in 3 patients associated with PZA due to transaminase elevation ( $n=3$, alanine aminotransferase were elevated to 2 times higher than the upper limit of the normal range) and gout ( $n=3) .4$ AEs occurrences in 2 patients associated with Pto due to transaminase elevation $(n=2$, alanine aminotransferase were elevated two times higher than the upper limit of the normal) and gastrointestinal reaction $(n=2)$. All AEs above were improved after discontinuation of the suspected drugs. $9 \otimes 60 \%, 9 / 15)$ of these patients returned to the discontinued drug after dosage adjustment and completed the course.

4 AEs in 2 patients $₫ 1.75 \% \varangle 2 / 114 \rrbracket$ were reported to be severe enough to require permanent discontinuation of the suspected drug: 1 case $(0.88 \% \otimes 1 / 114)$ was associated with Ak by hearing loss $(n=1)$ and mild renal insufficiency $(n=1)$. Allergic rash $(n=1)$ and gastrointestinal reaction $(n=1)$ caused by Mfx in 1 case $(0.88 \% \otimes 1 / 114)$. Patient with allergic skin rash were unable to tolerate and abandoned the treatment. 
As the rest 16 AEs occurrences in 13 patients $(11.40 \%, 13 / 114)$ were improved after symptomatic treatment, and no treatment plan was changed $\triangle 4 \mathrm{AEs}$ occurrences in 4 patients suspended Ak due to mild dizziness ( $n=4) ; 12$ AEs occurrencesin 9 patients suspended PZA and Pto due to mild transaminase elevation $(n=8$, alanine aminotransferase elevated 2 times lower than the upper limit of the normal range.) and gastrointestinal reaction $(n=4)$.

\section{Discussion}

In present study, we made up a regimen for the treatment on MDR-TB mainly based on guidelines of WHO 2016 and Chinese guidelines (15-17). We included MDR-TB patients with a low concentration INH resistance and without second-line drug/injection resistance according to MIC DST. The results showed that overall success rate was $79.8 \%$, in which treatment favorable rate reached as high as $91.7 \%$ in newly treated patients and $74 \%$ in retreated patients, the results demonstrated the high efficacy of this regimen against specific MDR-TB patients .

The Bangladesh short range regimen recommended by the 2016 guidelines is suggested for treating MDR patients without second-line drug resistance,which consisted of an intensive period of four to six months with seven drugs ( $\mathrm{Ak}, \mathrm{M}, \mathrm{Pt}, \mathrm{CfZ}, \mathrm{PZA}$, high-dose INH and EMB ) followed by a five-month course of $\mathrm{M}, \mathrm{CfZ}$, PZA and $E$ (18). But Cfz is expensive in China (about $\$ 400$ / month) and may be unacceptable to most Asian patients, especially young women, to accept due to the skin pigmentation. In present study, under inclusion conditions similar to the short range regimen: we replaced the high dose INH with $\mathrm{Pa}$ and replaced Cfz with Cs. High favorable treatment rate was also obtained and the entire treatment regimen costs about $\$ 300$ per month, while the short range regimen costs $\$ 450$ per month and the all-oral regimen recommended by the 2019 WHO guideline costs more than $\$ 2000$ per month in China. And the incidence of $\mathrm{AEs}$ in the present study is extremely low.

For resource-poor areas, the cost of treatment is an important determinant of patient compliance. Costly drugs, even if effective, may cause patients to discontinue treatment,which not only leads to the spread of drug-resistant MTB, but can also lead to more complex drug resistance in individuals. China is a high TB burden country with high financial burden of health care. Therefore, BDQ has not been widely used in China at present for its price. On the other hand, the WHO's clear recommendations on the use of LZD and BDQ could prove a double-edged sword for global TB control programmes (3). Intuitively, the advantages of including LZD and BDQ in standard protocols for all types of MDR-TB may be more conducive to programme implementation and less likely to require DST. The main drawback, however, may be concerns about patient safety and tolerability. Lzd is also expensive in China ( $\$ 600 /$ month) and has significant long-term side effects as an ultra-broad-spectrum antibiotic. About $30-40 \%$ of patients stop linezolid treatment because of AEs (19). Therefore, in present study, patients were strictly selected for inclusion, and only FQs was selected as the included drug among class A drugs.

The rate of $\mathrm{INH}$ acetylated is controlled by genetics. Once acetylated, $\mathrm{INH}$ is ineffective as an antibiotic against TB bacilli. Rapid acetylation of INH may lead to low serum concentrations of anti-TB drugs, 
increasing the risk of treatment failure. Most Asians are of the fast-metabolizing type (20). It had been reported that in the INH drug-resistant organisms studied, about $50 \%$ of INH MIC belong to the category of low concentration resistance with MIC at 0.1-1.0 ug / $\mathrm{mL}(21-23)$. A study from China showed that among $109 \mathrm{INH}$-resistant isolates, only $11.9 \%$ and $19.3 \%$ showed resistance to PAS and Pa, respectively (24).In present study, patients infected with low concentration INH resistant strains were included, and MIC values showed that all strains were sensitive to $\mathrm{Pa}$. Another advantage of $\mathrm{Pa}$ is its low price ( $\$ 22 \mathrm{per}$ month). Its safety was reflected in the incidence of $\operatorname{AEs}(0 \%)$.

Cycloserine(Cs)in group B has good antibacterial activity and the price in China is lower than Cfz. Due to its low drug resistance rate, low cross-resistance with other anti-TB drugs, and is oftem used as a good alternative drug $(25,26)$. The present study also verified its safety and efficacy.

Second-line injections (SLIs) were once one of the core drug groups in treatment of MDR-TB (15). However, existing studies have shown that SLIs has high side effects and often leads to withdrawal of patients (27). A retrospective study of 25 countries (28), according to the results of injection therapy (Ak) were better than no injections, but in analysis of comprehensive treatment results, patients who received the injection did worse than those who did not receive the injection. The results provided evidence for 2019 guidelines on use of injection drugs (1). But the article also explained that Ak may be the most widely used injectable drug due to its price and tended to be used in patients with the worst resistance patterns, which may be one of the reasons for poor outcomes rather than the problem with the drug itself. However, the present study showed that 18 AEs occurrences in $15(13.16 \%, 15 / 114)$ patients suspicious of Ak due to mild hearing loss or mild renal dysfunction. But most of them $(66,67 \%, 10 / 15)$ were treated with reduced doses. Only $1(6.67 \%, 1 / 15)$ patient had Ak permanently disabled. These results suggest that the Chinese population could be moderately tolerant to SLIs and that the cheap drug is certainly effective for certain populations.

\section{Conclusions}

The regimen in the present study had the following characteristics: with high curative effect, the favorable treatmentrate of MDR-TB patients with new treatment reached $91 \%$, and the patients were followed up for 1 year without recurrence; The use of $\mathrm{Pa}$ instead of high-dose INH and $\mathrm{Cs}$ instead of $\mathrm{Cfz}$ to treat specific MDR-TB population is more in line with China's national conditions; It proved that the AEs of SLIs are controllable in Chinese population and inexpensive; $72 \%$ (26/36) newly treated patients achieved the standard of stopping medicine at the end of of the 12th month. These characteristics suggest that the program could be widely used in China, even other resource-poor parts of Asia. Further research on the possibility of short-range treatment in resource-poor areas could be expected.

\section{Declarations}

\section{Ethical Approval and Consent to participate}


This study was approved by the Ethics Committee of Shanghai Pulmonary Hospital affiliated to Tongji University, the approval number was K16-298. Obtain written informed consent from each eligible MDRTB participant. An independent safety monitoring committee was established to ensure the continued safety of participants during clinical studies.

\section{Consent for publication}

Not applicable

\section{Availability of supporting data}

All data regarding the included participants and laboratory data during the study are available from the corresponding author by email request. The clinical study was registered at The China Clinical Trial Registry (ChiCTR, www.chictr.org.cn) with the registration number: ChiCTR-OPC-16009380.

\section{Competing interests}

The authors declare that they have no competing interests.

\section{Funding acknowledgements:}

This work was supported by the grant from the Shanghai Natural Science Foundation (Grant No.20ZR1446700). The funders had no role in study design, data collection and analysis, decision to publish, or preparation of the manuscript.

\section{Author contribution Statement}

WS: included the patients, treated and followed up the patients, wrote the manuscript; QT:included the patients, collected the data; JW and HY : culture isolated strain, $\mathbb{F Y}$ and JY凶Clinical laboratory for the work of MGIT 960 culture; LF: Research design, included the patients, data collection and revised the manuscript. All authors read and approved the final submitted version.

\section{Acknowledgements}

We thank all participants for their time and efforts.

\section{Author information:}

Affiliations

Shanghai Clinical Research Center for Tuberculosis, Shanghai Key Lab of Tuberculosis, Department of

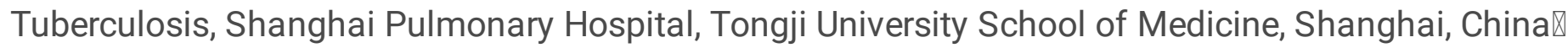
Wenwen Sun, Qin Tang and Lin Fan 
Shanghai Clinical Research Center for Tuberculosis, Shanghai Key Lab of Tuberculosis, Shanghai Pulmonary Hospital, Tongji University School of Medicine: Jie Wang and Hua Yang

Shanghai Clinical Research Center for Tuberculosis, Shanghai Key Lab of Tuberculosis, Department of clinical laboratory, Shanghai Pulmonary Hospital, Tongji University School of Medicine: Jinghui Yang, and Fangyou Yu

\section{Corresponding author}

Correspondence to Lin Fan

\section{References}

1. WHO. Global Tuberculosis Report 2019. Geneva, World health organization; 2019;2019(License):CCBY-NC-SA3.

2. WHO. WHO consolidated guidelines on drug-resistant tuberculosis treatment. world health organization. 2019;2019.

3. Chang K, Yew W. ATS/CDC/ERS/IDSA Clinical Practice Guidelines for Treatment of Drug-Resistant Tuberculosis: A Two-edged Sword? American journal of respiratory and critical care medicine. 2020;202(5):777-8.

4. Brouqui P, Quenard F, Drancourt M. Old antibiotics for emerging multidrug-resistant/extensively drugresistant tuberculosis (MDR/XDR-TB). International journal of antimicrobial agents. 2017;49(5):5547.

5. Zhang Y, Wu S, Xia Y, Wang N, Zhou L, Wang J, et al. Adverse Events Associated with Treatment of Multidrug-Resistant Tuberculosis in China: An Ambispective Cohort Study. Medical science monitor: international medical journal of experimental and clinical research. 2017;23:2348-56.

6. Falzon D, Schünemann H, Harausz E, González-Angulo L, Lienhardt C, Jaramillo E, et al. World Health Organization treatment guidelines for drug-resistant tuberculosis, 2016 update. The European respiratory journal. 2017;49(3).

7. Nunn A, Phillips P, Meredith S, Chiang C, Conradie F, Dalai D, et al. A Trial of a Shorter Regimen for Rifampin-Resistant Tuberculosis. The New England journal of medicine. 2019;380(13):1201-13.

8. Fan Y, Ding S, Bao Z, Wu L, Zhen L, Xia Q, et al. Prognostic factors for treatment success in patients with multidrug-resistant tuberculosis in China. The international journal of tuberculosis and lung disease : the official journal of the International Union against Tuberculosis and Lung Disease. 2018;22(3):300-5.

9. Ranzani O, Rodrigues L, Bombarda S, Minto C, Waldman E, Carvalho C. Long-term survival and cause-specific mortality of patients newly diagnosed with tuberculosis in São Paulo state, Brazil, 2010-15: a population-based, longitudinal study. The Lancet Infectious diseases. 2020;20(1):123-32.

10. Ismail N, Mvusi L, Nanoo A, Dreyer A, Omar S, Babatunde S, et al. Prevalence of drug-resistant tuberculosis and imputed burden in South Africa: a national and sub-national cross-sectional survey. 
The Lancet Infectious diseases. 2018;18(7):779-87.

11. Committee WHOGR. Guidelines for the programmatic management of drug-resistant tuberculosis2011 update. Geneva, Switzerland: World Health Organization, 2011. 2011.

12. Lange $C$, van Leth F, Mitnick C, Dheda K, Günther G. Time to revise WHO-recommended definitions of MDR-TB treatment outcomes. The Lancet Respiratory medicine. 2018;6(4):246-8.

13. Duan H, Chen X, Li Z, Pang Y, Jing W, Liu P, et al. Clofazimine improves clinical outcomes in multidrug-resistant tuberculosis: a randomized controlled trial. Clinical microbiology and infection : the official publication of the European Society of Clinical Microbiology and Infectious Diseases. 2019;25(2):190-5.

14. Diseases NloAal. Division of Microbiology and Infectious Diseases (DMID) adult toxicity table November 2007 draft 2017.

15. WHO. WHO treatment guidelines for drugresistant tuberculosis 2016 update. world health organization. 2016.

16. Association CA-t. the guideline of chemotherapy on MDR-TB in China. 2015;2015.

17. Association Ca-t. the guideline of chemotherapy on MDR-TB. 2019.

18. A VD, AK M, MA S, PK D, MR S, P D, et al. Short, highly effective, and inexpensive standardized treatment of multidrug-resistant tuberculosis. American journal of respiratory and critical care medicine. 2010;182(5):684-92.

19. Jaspard M, Butel N, El Helali N, Marigot-Outtandy D, Guillot H, Peytavin G, et al. Linezolid-Associated Neurologic Adverse Events in Patients with Multidrug-Resistant Tuberculosis, France. Emerging infectious diseases. 2020;26(8):1792-800.

20. Jagielski T, Bakuła Z, Roeske K, Kamiński M, Napiórkowska A, Augustynowicz-Kopeć E, et al. Mutation profiling for detection of isoniazid resistance in Mycobacterium tuberculosis clinical isolates. The Journal of antimicrobial chemotherapy. 2015;70(12):3214-21.

21. Parkin D, Vandenplas S, Botha F, Vandenplas M, Seifart H, van Helden P, et al. Trimodality of isoniazid elimination: phenotype and genotype in patients with tuberculosis. American journal of respiratory and critical care medicine. 1997;155(5):1717-22.

22. Nagel S, Streicher E, Klopper M, Warren R, Van Helden P. Isoniazid Resistance and Dosage as Treatment for Patients with Tuberculosis. Current drug metabolism. 2017;18(11):1030-9.

23. Van Deun A, Maug A, Salim M, Das P, Sarker M, Daru P, et al. Short, highly effective, and inexpensive standardized treatment of multidrug-resistant tuberculosis. American journal of respiratory and critical care medicine. 2010;182(5):684-92.

24. Li G, Zhang J, Jiang Y, Zhao L, Liu H, Li M, et al. Cross-resistance of isoniazid, para-aminosalicylic acid and pasiniazid against isoniazid-resistant Mycobacterium tuberculosis isolates in China. Journal of global antimicrobial resistance. 2020;20:275-81.

25. Wang J, Pang Y, Jing W, Chen W, Guo R, Han X, et al. Efficacy and safety of cycloserine-containing regimens in the treatment of multidrug-resistant tuberculosis: a nationwide retrospective cohort 
study in China. Infection and drug resistance. 2019;12:763-70.

26. Li Y, Wang F, Wu L, Zhu M, He G, Chen X, et al. Cycloserine for treatment of multidrug-resistant tuberculosis: a retrospective cohort study in China. Infection and drug resistance. 2019;12:721-31.

27. Shean K, Streicher E, Pieterson E, Symons G, van Zyl Smit R, Theron G, et al. Drug-associated adverse events and their relationship with outcomes in patients receiving treatment for extensively drugresistant tuberculosis in South Africa. PloS one. 2013;8(5):e63057.

28. J Peter Cegielski, Pei-Chun Chan, Zhiyi Lan,et al.Aminoglycosides and Capreomycin in the Treatment of Multidrug-resistant Tuberculosis: Individual Patient Data Meta-analysis of 12030 Patients From 25 Countries, 2009-2016, Clinical Infectious Diseases, 2020, ciaa621

\section{Tables}

Table 1 Clinical characteristics of patients enrolled

\begin{tabular}{|llll|}
\hline characteristic & Newly treated $(\mathrm{n}=36)$ & $\begin{array}{l}\text { Retreated } \\
(\mathrm{n}=78)\end{array}$ & P value \\
\hline median age & $32.1 \pm 12.4$ & $35.5 \pm 11.5$ & 0.13 \\
\hline Male (\%) & $22(61 \%)$ & $50(64.1 \%)$ & 0.34 \\
\hline median BMI & 19.8 & 19.2 & 0.54 \\
\hline cavities present on Chest CT & & & \\
\hline no cavity & $18(50 \%)$ & $36(46.2 \%)$ & 0.82 \\
\hline unilateral & $12(33.3 \%)$ & $35(44.9 \%)$ & 0.08 \\
\hline bilateral & $5(13.9 \%)$ & $7(9.0 \%)$ & 0.14 \\
\hline lesion severity & & & \\
\hline$\geq 3$ fields & $22(61.1 \%)$ & $49(62.8 \%)$ & 0.55 \\
\hline$<3$ fields & $14(38.9 \%)$ & $29(37.2 \%)$ & 0.76 \\
\hline complications & & $10(12.8 \%)$ & 0.35 \\
\hline DM & $5(13.9 \%)$ & & \\
\hline
\end{tabular}

Table 2 The differences of time to culture conversion between two groups 


\begin{tabular}{|llll|}
\hline & $\begin{array}{l}\text { Newly } \\
\text { treated } \\
(n=36)\end{array}$ & $\begin{array}{l}\text { Retreated } \\
(n=78)\end{array}$ & $\begin{array}{l}P \\
\text { value }\end{array}$ \\
\hline sputum negative conversion rate at month 2 $(n, \%)$ & $15(41.67)$ & $18(23.08)$ & 0.00 \\
\hline sputum negative conversion rate at month 3 (n,\%) & $33(91.67)$ & $54(69.23)$ & 0.01 \\
\hline sputum negative conversion rate at month 6 (n,\%) & $34(94.44)$ & $55(70.51)$ & 0.00 \\
\hline $\begin{array}{l}\text { Achieve the standard of stopping medicine at the end of 12 } \\
\text { month }\end{array}$ & $26(72)$ & $25(32)$ & 0.00 \\
\hline
\end{tabular}

\section{Figures}

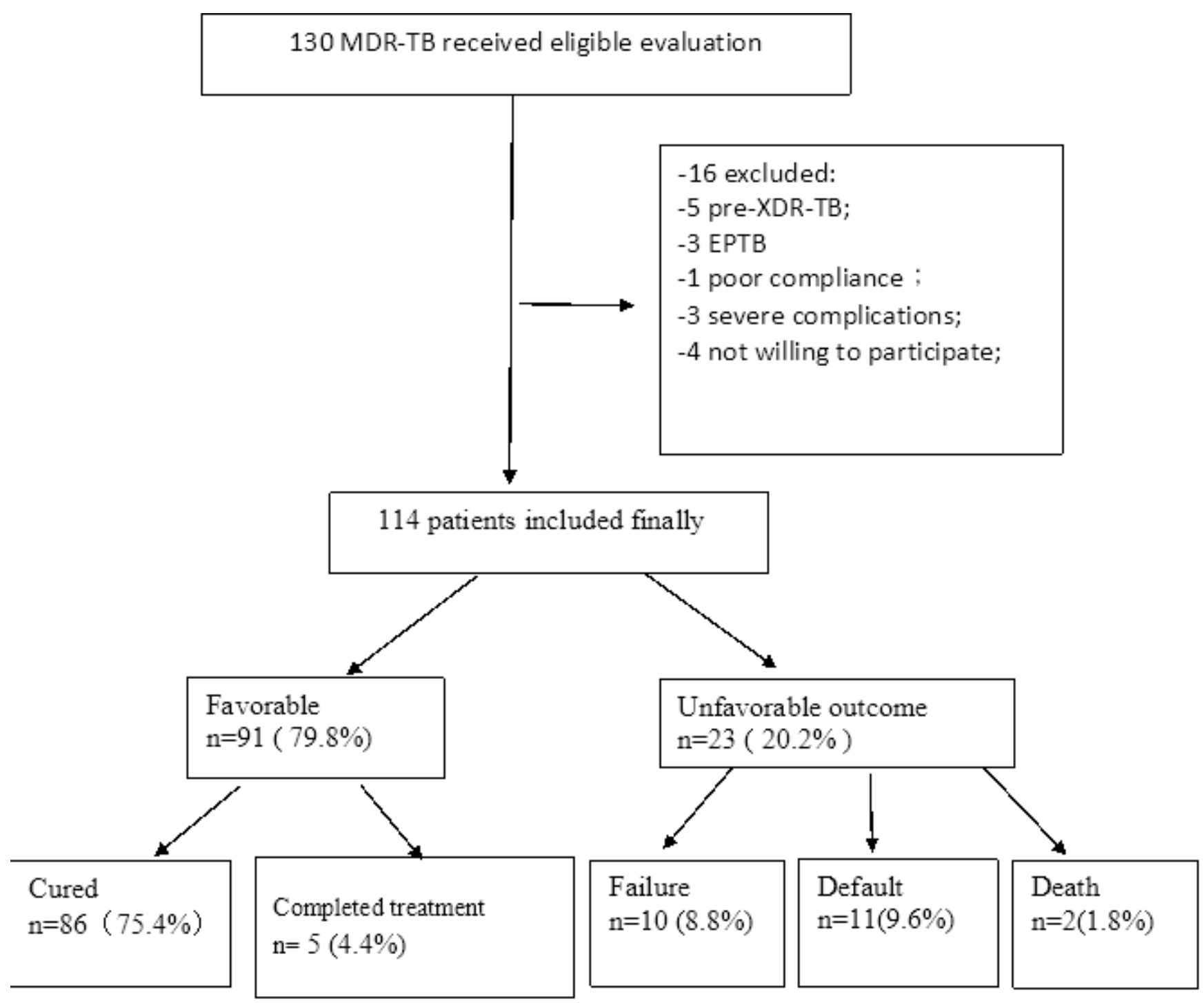

\section{Figure 1}

Flow diagram 
Time to culture conversion months between two groups

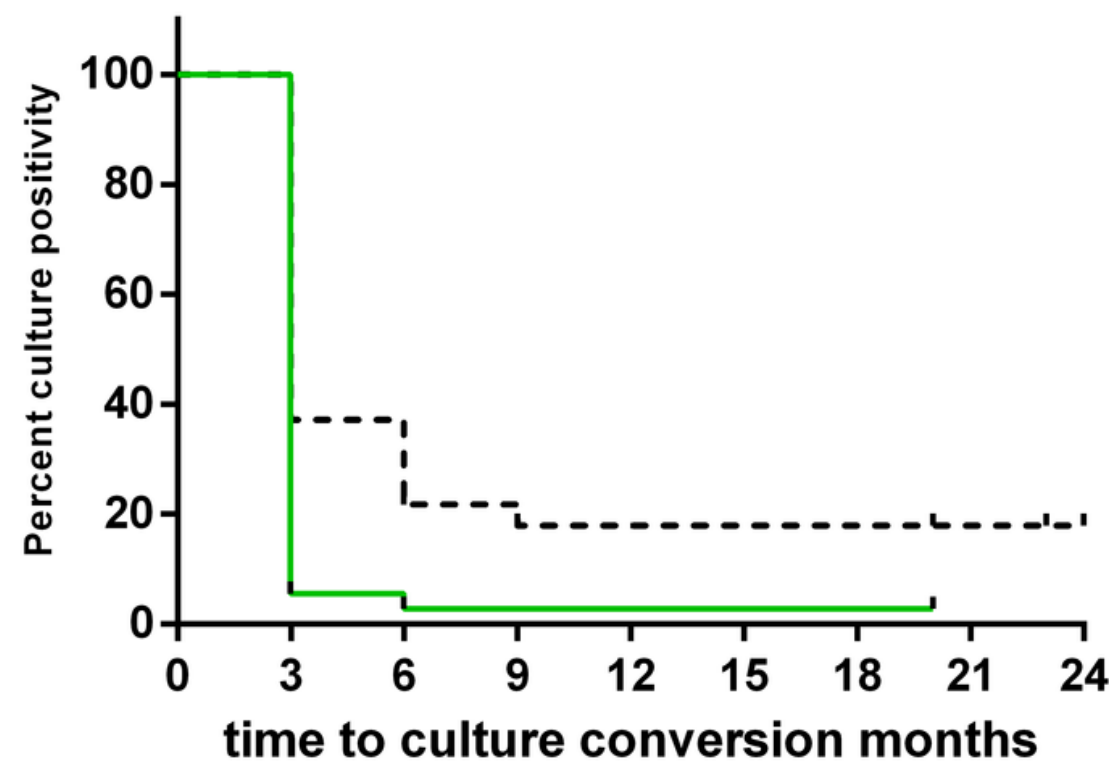

$\perp$ newly treated MDR-TB

- . retreated MDR-TB

Figure 2

Treatment outcome 